\title{
The Influence of Alcohol Consumption in Conjunction with Sex Hormone Deficiency on Ca/P Ratio in Rats
}

\author{
Karina Bortolin Lodi, ${ }^{1}$ Adriana Mathias Pereira da Silva Marchini, ${ }^{1}$ \\ Ana Maria do Espírito Santo, ${ }^{2,3}$ Sigmar de Mello Rode, ${ }^{4}$ \\ Leonardo Marchini, ${ }^{5}$ and Rosilene Fernandes da Rocha ${ }^{1}$ \\ ${ }^{1}$ Institute of Science and Technology, Department of Biosciences and Diagnostics, Universidade Estadual Paulista (UNESP), \\ Avenida Engenheiro Francisco José Longo 777, 12245-000 São José dos Campos, SP, Brazil \\ ${ }^{2}$ Institute of Environmental Science, Chemical and Pharmaceutical, Department of Mathematical and Earth Sciences, \\ Federal University of São Paulo (UNIFESP), Rua Prof. Artur Riedel 275, 09972-270 Diadema, SP, Brazil \\ ${ }^{3}$ Institute of Research and Development, University of Vale do Paraíba (UNIVAP), Laboratory of Biomedical Vibrational Spectroscopy, \\ Avenida Shishima Hifumi 2911, 12244-000 São José dos Campos, SP, Brazil \\ ${ }^{4}$ Institute of Science and Technology, Department of Dental Materials and Prosthesis, Universidade Estadual Paulista (UNESP), \\ Avenida Engenheiro Francisco José Longo 777, 12245-000 São José dos Campos, SP, Brazil \\ ${ }^{5}$ Department of Preventive and Community Dentistry, The University of Iowa College of Dentistry and Dental Clinics, \\ N337-1 Dental Science N, 52242 Iowa City, IA, USA
}

Correspondence should be addressed to Adriana Mathias Pereira da Silva Marchini; adrianampsmarchini@gmail.com

Received 12 October 2015; Revised 16 February 2016; Accepted 22 February 2016

Academic Editor: Marek Bolanowski

Copyright (C) 2016 Karina Bortolin Lodi et al. This is an open access article distributed under the Creative Commons Attribution License, which permits unrestricted use, distribution, and reproduction in any medium, provided the original work is properly cited.

Deficiency of sex hormones and excessive alcohol consumption are factors that have been related to alterations in the pattern of bone mineralization and osteoporosis. The aim of this study was to evaluate possible alterations in the calcium/phosphorus (Ca/P) ratio in the femur of rats subjected to sex hormone deficiency and/or alcohol consumption. Methods. Female and male Wistar rats $(n=108)$ were divided into ovariectomized (Ovx), orchiectomized (Orx), or sham-operated groups and subdivided according to diet: alcoholic diet ( $20 \%$ alcohol solution), isocaloric diet, and ad libitum diet. The diets were administered for 8 weeks. The Ca/P ratio in the femur was analyzed by energy dispersive micro-X-ray spectrometer ( $\mu$ EDX). Results. Consumption of alcohol reduced the $\mathrm{Ca} / \mathrm{P}$ ratio in both females and males. The isocaloric diet reduced the $\mathrm{Ca} / \mathrm{P}$ ratio in females. In groups with the ad libitum diet, the deficiency of sex hormones did not change the $\mathrm{Ca} / \mathrm{P}$ ratio in females or males. However, the combination of sex hormone deficiency and alcoholic diet presented the lowest values for the $\mathrm{Ca} / \mathrm{P}$ ratio in both females and males. Conclusions. There was a reduced $\mathrm{Ca} / \mathrm{P}$ ratio in the femur of rats that consumed alcohol, which was exacerbated when combined with a deficiency of sex hormones.

\section{Introduction}

Bone is a specialized form of connective tissue formed by minerals, organic matrix, water, and lipids. Calcium $(\mathrm{Ca})$ and phosphorus $(\mathrm{P})$ are the most important constituent elements of the bone mineral phase, forming hydroxyapatite crystals $\left[\mathrm{Ca}_{10}\left(\mathrm{PO}_{4}\right)_{6}(\mathrm{OH})_{2}\right]$. Compared with geological hydroxyapatite, the bone crystals are small, poorly crystalline, and more soluble. This solubility allows the maintenance of mineral homeostasis, since the $\mathrm{Ca}$ and $\mathrm{P}$ also have important metabolic functions $[1,2]$. In adults, the degree of mineralization depends, primarily, on changes in the bone remodeling process. This process is characterized by constant activity of osteoclasts (bone resorbing cells) and osteoblasts (bone forming cells). Diseases that change the pattern of bone turnover, such as osteoporosis, can modify the degree of bone 
mineralization as well as the chemical composition of the hydroxyapatite, changing $\mathrm{Ca}$ and $\mathrm{P}$ proportions $[3,4]$.

Osteoporosis can be classified as primary and secondary. In clinical practice, the most common forms of primary osteoporosis are those that affect women after menopause (type I) and age-related osteoporosis that affects both women and men (type II) $[5,6]$.

Deficiency of sex hormones has an important role in the development of osteoporosis. This deficiency is well-known to affect women after menopause. However, osteoporosis caused by sex hormone deficiency can also affect men. In males, the serum levels of testosterone (the main sex steroid regulating bone metabolism in men) gradually decrease with age. This leads to decreased levels of estrogen (since estrogen can be synthesized from testosterone). Both estrogen and testosterone are important in maintaining bone health; however, estrogen deficiency seems to be the most important determinant of bone loss associated with age in both men and women [7-10]. It is believed that estrogen deficiency causes changes in bone remodeling due primarily to an increase in the resorption process, although a decrease in bone formation is also described $[6,10,11]$.

Osteoporosis can also present secondary causes related mainly to lifestyle, nutritional abnormalities, use of medicines, and endocrine/metabolic disorders. Secondary causes of osteoporosis could increase bone loss and fracture risk, especially in the elderly $[5,6]$. Chronic abuse of alcohol is also widely cited as a cause of secondary osteoporosis $[12,13]$. The decrease in bone mass related to the consumption of alcohol seems to be related to bone remodeling alterations, with a predominant decrease in bone formation, as alcohol has been considered toxic for osteoblasts [13, 14].

Alcohol consumption can also cause significant nutritional changes. In part, this happens because alcohol has low nutritional value but high energy content, which may lead the consumer to replace the intake of nutritive food with alcohol. Moreover, consumption of alcohol leads to malabsorption and maldigestion of food [15]. Since adequate nutrient intake is important for maintaining bone quality [16], this factor should be considered in studies aiming to evaluate the influence of alcohol on bone tissue. Alcohol consumption is also related to cognitive disorders that lead to an increased incidence of falls and bone fractures, which can increase levels of morbidity and mortality in patients with osteoporosis [17].

Considering the importance of $\mathrm{Ca}$ and $\mathrm{P}$ on bone chemical composition, and the negative influence of estrogen/testosterone deficiency and/or alcohol consumption on bone quality, the objective of this study is to evaluate possible changes in the quantity of $\mathrm{Ca}$ and $\mathrm{P}$ in the femur of ovariectomized (Ovx) or orchiectomized (Orx) rats receiving an alcoholic diet.

In previously published studies, adverse effects on bone quality were observed in female rats when alcohol was associated with estrogen deficiency $[18,19]$, although few data exist about what occurs in males. Based on the results of these studies, our hypothesis is that deficiencies in sex hormones associated with alcohol consumption can cause a change in bone mineralization with a reduced $\mathrm{Ca} / \mathrm{P}$ ratio.

\section{Methods}

2.1. Surgical Procedures, Diet, and Weight. This study was performed in accordance with the ethical standards adopted in animal experimentation and was approved by the Ethics Committee of São José dos Campos School of Dentistry, Universidade Estadual Paulista (UNESP) (protocol number 037/2007-PA/CEP).

One hundred and eight rats (Rattus norvegicus, albino, Wistar) at a 1:1 male/female ratio, aged four months, were initially divided into Ovx, Orx, or sham-operated (Sham) groups. In the Ovx group, the ovaries were removed; in the Orx group, the testicles were removed. The Sham groups were subjected to a surgical procedure similar to the other groups in terms of anesthesia and surgically exposing the ovaries or testicles, but the ovaries or testicles were not removed. The purpose of this procedure was to control for the possible influence of surgical stress on the results. With this method, the Ovx and Orx groups were subjected to a deficiency of sex hormones and the Sham groups were the experimental control.

One month after the surgery, animals were subdivided into alcoholic, isocaloric, and ad libitum dietary groups. The interval of one month between the surgical procedure and the beginning of the diet allows sufficient time for the animal to recover from the stress of the surgical procedure, allowing a good adaptation to dietary changes. This same interval was previously described in other studies [19-22]. In the alcoholic diet group, animals received a $20 \%$ alcohol solution as the only source of liquids (freely, until they were satiated) and a commercial solid food (freely, until they were satiated). In the isocaloric diet group, animals received water (freely, until they were satiated), $26.6 \%$ sucrose solution (in restricted quantity), and commercial solid food (in restricted quantity). The amount of sucrose solution and solid food offered to animals that received the isocaloric diet contained the same amount of calories ingested by animals with the alcoholic diet the day before (pair-fed diet). In doing this, the animals with the isocaloric diet started the diet a day after the animals with the alcoholic diet. In the ad libitum diet, the animals received water (freely, until they were satiated) and commercial solid food (freely, until they were satiated).

In summary, the animals were divided into 12 experimental groups with 9 animals in each $(n=9)$ as described in Table 1. More details regarding the methodology of treatment of animals have been previously published [19]. In this study [19], the authors used the same experimental protocol and treatment group subdivision as described herein, but only for female rats: deficiency of sex hormones (Ovx) associated with alcoholic diet (20\% alcohol solution), isocaloric diet, or ad libitum diet.

For all groups, the average values of daily intake for solid foods and liquids were recorded. The weight changes (\%) were also measured for each animal using the difference between final weight $(\mathrm{g})$ and initial weight $(\mathrm{g})$. The value obtained was then divided by the initial weight and multiplied by 100 . The initial weight was measured on day of surgery (Ovx, Orx, or Sham) and the final weight on the day of sacrifice. 
TABLE 1: Description of the experimental groups.

\begin{tabular}{|c|c|c|c|c|}
\hline Group number & Group name (short form) & Gender & Surgery & Diet \\
\hline G1 & f-ovx-alc & Female & Ovariectomy & Alcoholic \\
\hline G2 & f-ovx-iso & Female & Ovariectomy & Isocaloric \\
\hline G3 & f-ovx-ad & Female & Ovariectomy & Ad libitum \\
\hline G4 & f-sham-alc & Female & Sham & Alcoholic \\
\hline G5 & f-sham-iso & Female & Sham & Isocaloric \\
\hline G6 & f-sham-ad & Female & Sham & Ad libitum \\
\hline G7 & m-orx-alc & Male & Orchiectomy & Alcoholic \\
\hline G8 & m-orx-iso & Male & Orchiectomy & Isocaloric \\
\hline G9 & m-orx-ad & Male & Orchiectomy & Ad libitum \\
\hline G10 & m-sham-alc & Male & Sham & Alcoholic \\
\hline G11 & m-sham-iso & Male & Sham & Isocaloric \\
\hline G12 & m-sham-ad & Male & Sham & Ad libitum \\
\hline
\end{tabular}

To analyze the capacity to convert the energy consumed into body weight, a calculation of the feed efficiency ratio was performed for each animal: the weight gain (g) was divided by total dietary intake (kcal) and multiplied by 100 .

At the beginning of dietary treatment, which began one month after surgery, animals with the alcoholic diet underwent an adaptation period of 9 days, in which they received alcohol solutions at gradually increasing concentrations: 3 days receiving $5 \%$ alcohol solution, 3 days receiving $10 \%$ alcohol solution, and 3 days receiving $15 \%$ alcohol solution. This period of adaptation decreases a possible aversion to sudden dietary change, allowing greater acceptance of treatment. A similar adaptation protocol (the same timeframe and alcohol concentrations) has been described previously [20].

After this adaptation period, the alcohol groups began to receive the alcoholic solution at $20 \%$, and the isocaloric groups began to receive the calorie-restricted diet. For the ad libitum groups there were no changes in the diet. These diets were maintained for 8 weeks. The daily measurement of the amount of food and liquid intake (to calculate caloric intake, feed efficiency, and water consumption) began only after the $20 \%$ concentration had been established. At the end of dietary treatment, the animals were sacrificed.

All animals were anesthetized before surgical procedures (Ovx, Orx, or Sham) and sacrifices. An intramuscular injection of a xylazine chloride $(2.3 \mathrm{~g} / 100 \mathrm{~mL})$ and ketamine chloride $(1.16 \mathrm{~g} / 10 \mathrm{~mL})$ solution was used for this purpose. Initially, a master solution was prepared with $0.8 \mathrm{~mL}$ of xylazine chloride and $0.5 \mathrm{~mL}$ of ketamine chloride. To promote general anesthesia, the animals received $0.1 \mathrm{~mL}$ of this solution for each $100 \mathrm{~g}$ of body weight.

\subsection{Energy Dispersive Micro-X-Ray Fluorescence Spectroscopy.} The right femurs were dissected and stored in a freezer $\left(-80^{\circ} \mathrm{C}\right)$. They were evaluated by an energy dispersive micro$\mathrm{X}$-ray spectrometer $(\mu \mathrm{EDX})$ to determine the concentration of $\mathrm{Ca}(\%)$ and $\mathrm{P}(\%)$, enabling the calculation of the $\mathrm{Ca} / \mathrm{P}$ ratio. The analyses were performed in equipment with a rhodium X-ray tube (Model 1300 Shimadzu $^{\circledR}$, Kyoto, Japan). The radiation count was made by a silicon lithium semiconductor detector cooled by liquid nitrogen and connected to a computer for data processing.

The X-ray fluorescence spectroscopy was performed as a semiquantitative analysis. A similar methodology was previously published $[23,24]$. The present study fixed the fundamental parameters for $\mathrm{Ca}$ and $\mathrm{P}$ using commercial stoichiometric hydroxyapatite (Aldrich, synthetic $\mathrm{Ca}_{10}\left(\mathrm{PO}_{4}\right)_{6}(\mathrm{OH})_{2}$, grade $99.999 \%$, lot $10818 \mathrm{H})$. The chemical elements carbon $(\mathrm{C})$, oxygen $(\mathrm{O})$, and hydrogen $(\mathrm{H})$ were set as balance (since the bone tissue consists of a carbonated hydroxyapatite). The total mass proportion of the sample $(100 \%)$ was calculated as $x+y+z$ (where $x=\mathrm{Ca}, y=\mathrm{P}$, and $z=\mathrm{C}+\mathrm{O}+\mathrm{H})$. Only Ca and $\mathrm{P}$ were directly measured by the equipment. The organic elements $(\mathrm{C}, \mathrm{O}$, and $\mathrm{H})$ were not directly measured because the equipment used in this study does not perform data for elements below sodium $(\mathrm{Na})$ in atomic number. Other inorganic elements as magnesium $(\mathrm{Mg}), \mathrm{Na}$, and potassium (K) were not identified in any spectra, indicating they were absent in the samples or their amounts were below the limit of detection.

Equipment voltage was set at $15 \mathrm{kV}$ with automatic adjustment of the current and beam diameter of $50 \mu \mathrm{m}$. The equipment allows drawing a line over which the sample can be analyzed. In this study, the analysis was performed on a horizontal line, which was drawn across the longitudinal length of the bone in the central part (excluding the epiphyses, which were previously cut). The chosen surface is relatively flat, which is important for a proper analysis when using this type of technique. The analyses were performed each $50 \mu \mathrm{m}$ of bone length, totaling 200 points per sample with a reading time of 10 seconds/real time per point.

After finishing the reading, a graph was generated with the positioning and the mean of the $\mathrm{Ca}$ and $\mathrm{P}$ concentrations (\%). The ratio of the chemical elements in the sample was calculated as the ratio of the relative weight given in the readings of the device, which converts the relative intensity of the characteristic transition energy into element content by weight percentage.

For this study, we considered that the synthetic hydroxyapatite, $\mathrm{Ca}_{10}\left(\mathrm{PO}_{4}\right)_{6}(\mathrm{OH})_{2}$, consists of about $39.90 \%$ of $\mathrm{Ca}$, $18.50 \%$ of $\mathrm{P}, 41.41 \%$ of $\mathrm{O}$, and $0.20 \%$ of $\mathrm{H}$ (these values were 
obtained by stoichiometric calculations from the number of atoms and atomic mass of each chemical element present in the molecular formula of hydroxyapatite). Calculations of $\mathrm{Ca} / \mathrm{P}$ ratio were made considering the number of atoms of $\mathrm{Ca}$ and $\mathrm{P}$ (10 and 6, resp.) and the relative percentages of $\mathrm{Ca}$ and $\mathrm{P}(39.90 \%$ and $18.50 \%$, resp.) present in the hydroxyapatite molecule, using the following formula:

$$
\frac{\mathrm{Ca}}{\mathrm{P}}=\frac{(10 \times c) \div 39.90}{(6 \times p) \div 18.50} \text {. }
$$

In the above formula, the letter $c$ represents the value of $\mathrm{Ca}$ (\%) and the letter $p$ represents the value of $\mathrm{P}(\%)$. The values of $\mathrm{Ca}(\%)$ and $\mathrm{P}(\%)$ were obtained in the analysis of the samples.

2.3. Statistical Analysis. Dietary analysis and feed efficiency were tabulated and presented. Data related to the analysis of $\mathrm{Ca}(\%), \mathrm{P}(\%)$, and $\mathrm{Ca} / \mathrm{P}$ were evaluated by one-way ANOVA and Tukey's test. Data related to changes in weight (\%) showed nonnormal distribution and were performed using nonparametric test (Kruskal-Wallis followed by DunnBonferroni). A significance level of 0.05 (5\%) was adopted for all tests.

The program Minitab ${ }^{\circledR}$ (version 15.1.1.0 Minitab Inc., State College, PA, USA) was used for statistical analysis. The figures were made using the program Microsoft Excel 2007 (Microsoft Corporation, Redmond, WA, USA).

\section{Results}

3.1. Overall Remarks. In animals subjected to the alcoholic diet, a lethargic state was observed, characterized by excessive resting (probably as a result of alcohol consumption). In the Ovx and Orx groups, the atrophy of the uterus and seminal vesicles was noticed after sacrifice.

3.2. Weight Changes (\%). The values of the averages and standard deviations for weight changes (\%) are illustrated in Figure 1. The statistical comparisons for weight changes (\%) are summarized in the legend of Figure 1.

In the analysis of weight changes (\%), it was observed that all females gained weight during the experiment. The group which gained more weight was G3, representing females with estrogen deficiency and ad libitum diet. This group (G3) was statistically different from groups G1 (f-ovx-alc), G2 (f-ovxiso), G4 (f-sham-alc), and G5 (f-sham-iso). This means that group G3 (f-ovx-ad) was statistically different from all groups with controlled diets (alcoholic or isocaloric).

Regarding the males, animals in groups G7 (m-orx-alc), G8 (m-orx-iso), and G11 (m-sham-iso) lost weight during the experiment. Conversely, males in the other groups gained weight. Males who gained most weight were those with the ad libitum diet (G9 and G12). These groups (G9 and G12) were statistically different from G7 (m-orx-alc), G8 (m-orx-iso), and G11 (m-sham-iso).

3.3. Caloric Intake, Feed Efficiency, and Water Consumption. For females, the highest values of caloric intake $(66.19 \mathrm{kcal})$

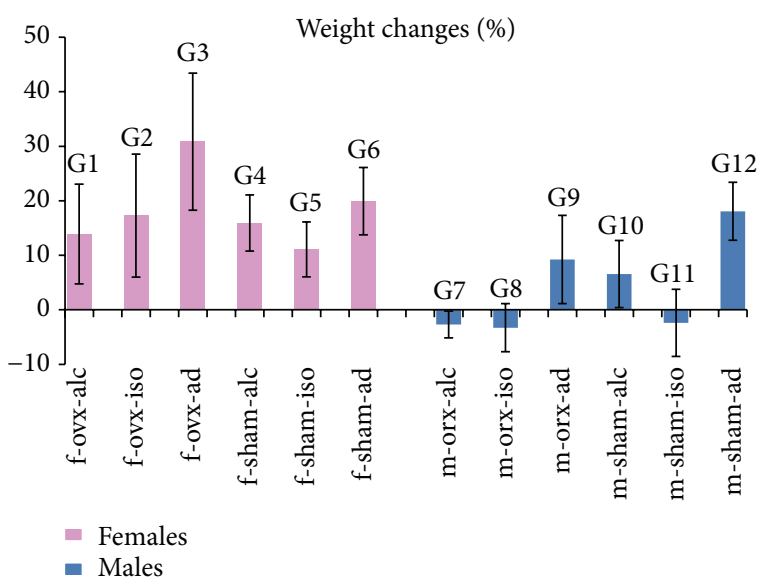

FIGURE 1: Weight changes (\%): the graph illustrates the values of the averages (bars) and standard deviations (lines superimposing the bars). Statistical comparisons are summarized as follows: females: comparison between most of the groups showed no significant differences (NS). Statistical significances $(p<0.05)(*)$ were observed in the following groups: G1/G3 (*); G2/G3 (*); G4/G3 (*); G5/G3 (*). Males: comparison between most of the groups showed no significant differences (NS). Statistical significances $(p<0.05)$ $(*)$ were observed in the following groups: G9/G7 (*); G9/G8 (*); G9/G11 (*); G12/G7 (*); G12/G8 (*); G12/G11 (*).

and feed efficiency (46.61\%) were observed in G3 (f-ovx$\mathrm{ad)}$. On the other hand, the lowest values of caloric intake (57.76 kcal) and feed efficiency (19.22\%) were observed in G5 (f-sham-iso). It is interesting to observe that, in females on an alcoholic diet, G1 (f-ovx-alc) and G4 (f-sham-alc), relatively high caloric intake $(64.31 \mathrm{kcal}$ and $65.70 \mathrm{kcal})$ but relatively low feed efficiency $(21.61 \%$ and $24.25 \%)$ was observed (Table 2).

Regarding males, feed efficiency values were proportional to calorie intake for all groups: when caloric intake increased, the feed efficiency has also increased. The highest values of caloric intake $(105.83 \mathrm{kcal})$ and feed efficiency $(17.08 \%)$ were found in G12 (m-sham-ad). On the other hand, the lowest values of caloric intake $(69.32 \mathrm{kcal})$ and feed efficiency $(-4.73 \%)$ were found in G8 (m-orx-iso) (Table 2 ).

For females, the lowest water consumption occurred in animals receiving the alcoholic diet, G1 $(12.99 \mathrm{~mL})$ and $\mathrm{G} 4$ $(13.71 \mathrm{~mL})$. Similarly, for males, the lowest water consumption also occurred in animals receiving the alcoholic diet, G7 $(16.57 \mathrm{~mL})$ and G10 $(15.70 \mathrm{~mL})$ (Table 2). It should be emphasized that the calculations for water consumption for animals receiving the alcoholic diet considered the water that the alcohol was dissolved in, and these animals did not receive another form of hydration.

3.4. Analysis of $\mathrm{Ca}$ (\%). The values of the averages and standard deviations for $\mathrm{Ca}(\%)$ are illustrated in Figure 2. The statistical comparisons for $\mathrm{Ca}(\%)$ are summarized in the legend of Figure 2.

Regarding females, the highest value for $\mathrm{Ca}$ (\%) was obtained in G3 (f-ovx-ad), and the lowest value was obtained in $\mathrm{Gl}$ (f-ovx-alc). It is interesting to note that both groups 
TABLE 2: Total caloric intake, alcohol intake, water, and feed efficiency.

\begin{tabular}{lcccc}
\hline Group & Total caloric intake (kcal) & Alcohol intake (kcal) & Water $(\mathrm{mL})$ & Feed efficiency $(\%)$ \\
\hline G1 (f-ovx-alc) & 64.31 & 18.40 & 12.99 & 27.13 \\
G2 (f-ovx-iso) & 58.31 & - & 25.0 & 29.65 \\
G3 (f-ovx-ad) & 66.19 & 19.41 & 13.71 & 46.61 \\
G4 (f-sham-alc) & 65.70 & - & 28.24 & 24.25 \\
G5 (f-sham-iso) & 57.76 & - & 32.00 & 19.22 \\
G6 (f-sham-ad) & 60.37 & 20.71 & 16.57 & 33.03 \\
G7 (m-orx-alc) & 70.11 & - & 36.19 & -3.85 \\
G8 (m-orx-iso) & 69.32 & - & 39.70 & -4.73 \\
G9 (m-orx-ad) & 90.34 & 19.63 & 15.70 & 3.11 \\
G10 (m-sham-alc) & 80.93 & - & 33.90 & -3.33 \\
G11 (m-sham-iso) & 72.17 & - & 43.04 & 17.08 \\
G12 (m-sham-ad) & 105.83 & & - & \\
\hline
\end{tabular}

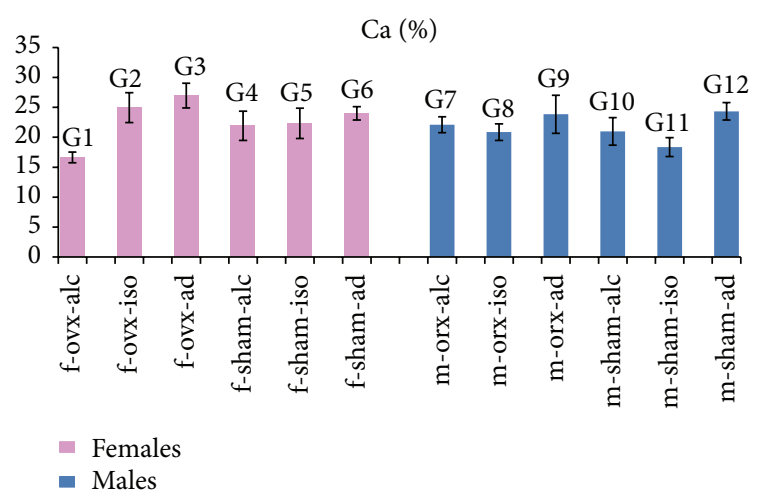

Figure 2: Ca (\%): the graph illustrates the values of the averages (bars) and standard deviations (lines superimposing the bars). Statistical comparisons are summarized as follows: females: comparison between most of the groups showed statistical significance $(p<0.05)$. No significant differences (NS) were observed in the following groups: G2/G6 (NS); G4/G5 (NS). Males: comparison between most of the groups showed statistical significance $(p<$ $0.05)$. No significant differences (NS) were observed in the following groups: G9/G12 (NS); G10/G7 (NS); G10/G8 (NS).

(G3 and G1) were submitted to hormonal deficiency, so the difference was in the type of the diet (ad libitum or alcoholic). Statistically significant differences were observed among most of the females groups. However, no difference was observed between the following groups: G2 (f-ovx-iso) and G6 (f-sham-ad); G4 (f-sham-alc) and G5 (f-sham-iso).

Regarding males, the highest value for $\mathrm{Ca}$ (\%) was obtained in G12 (m-sham-ad), and the lowest value was obtained in G11 (m-sham-iso). It is interesting to note that neither group (G12 and G11) had hormone deficiency, so the difference was the type of diet (ad libitum or isocaloric). Statistical differences were observed among most of the males groups. However, no difference was observed between the following groups: G9 (m-orx-ad) and G12 (m-sham-ad); G10 (m-sham-alc) and G7 (m-orx-alc); G10 (m-sham-alc) and G8 (m-orx-iso).

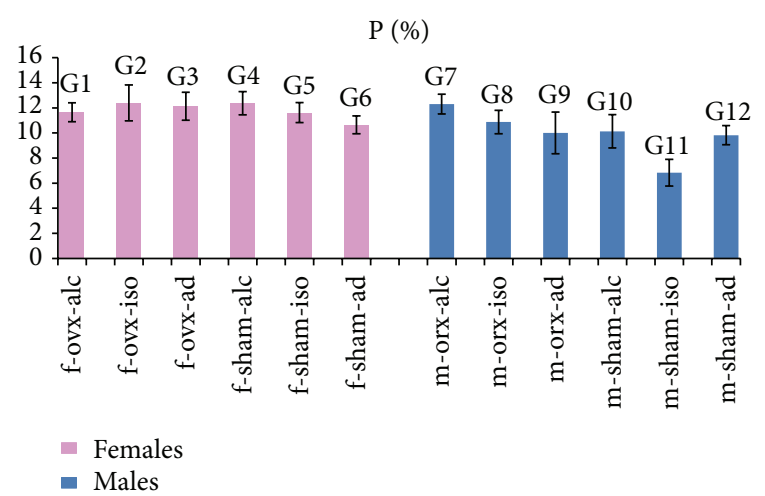

Figure 3: P (\%): the graph illustrates the values of the averages (bars) and standard deviations (lines superimposing the bars). Statistical comparisons are summarized as follows: females: comparison between most of the groups showed statistical significance $(p<0.05)$. No significant differences (NS) were observed in the following groups: G1/G5 (NS); G2/G3 (NS); G2/G4 (NS), G3/G4 (NS). Males: comparison between most of the groups showed statistical significance $(p<0.05)$. No significant differences (NS) were observed between the following groups: G10/G12 (NS); G9/G10 (NS); G9/G12 (NS).

3.5. Analysis of $P(\%)$. The values of the averages and standard deviations for $\mathrm{P}(\%)$ are illustrated in Figure 3. The statistical comparisons for $\mathrm{P}(\%)$ are summarized in the legend of Figure 3.

Regarding females, the highest value for $\mathrm{P}$ (\%) was obtained in G2 (f-ovx-iso), and the lowest value was obtained in G6 (f-sham-ad). Statistically significant differences were observed among most of the females groups. However, no difference was observed between the following groups: G1 (fovx-alc) and G5 (f-sham-iso); G2 (f-ovx-iso) and G3 (f-ovxad); G2 (f-ovx-iso) and G4 (f-sham-alc); G3 (f-ovx-ad) and G4 (f-sham-alc).

Regarding males, the highest value for $\mathrm{P}(\%)$ was obtained in G7 ( $\mathrm{m}$-orx-alc), and the lowest value was obtained in G11 (m-sham-iso). Statistically significant differences were 


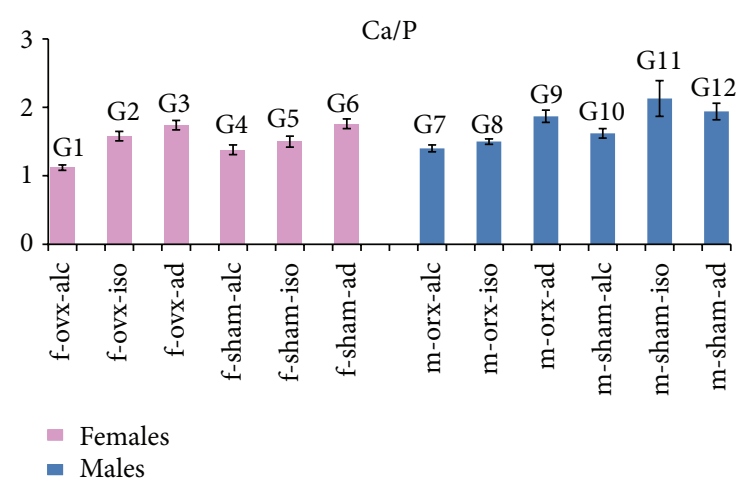

FIGURE 4: Ca/P: the graph illustrates the values of the averages (bars) and standard deviations (lines superimposing the bars). Statistical comparisons are summarized as follows: females: comparison between most of the groups showed statistical significance $(p<$ 0.05 ). No significant differences (NS) were observed in the following groups: G3/G6 (NS). Males: comparison between most of the groups showed statistical significance $(p<0.05)$. No significant differences (NS) were observed in the following groups: G12/G9 (NS); G12/G11 (NS).

observed among most of the males groups. However, no difference was observed in the comparisons between the following groups: G10 (m-sham-alc) and G12 (m-sham-ad); G9 (m-orx-ad) and G10 (m-sham-alc); G9 (m-orx-ad) and G12 (m-sham-ad).

It is interesting to note that males subjected to hormonal deficiency and alcoholic diet (G7) demonstrated the highest values of $\mathrm{P}(\%)$, compared to other groups of males. In contrast, of females subjected to the comparable experimental protocol, G1 (f-ovx-alc) showed a small reduction in P (\%) relative to most of the other female groups.

3.6. Analysis of $\mathrm{Ca} / \mathrm{P}$ Ratio. The values of the averages and standard deviations for $\mathrm{Ca} / \mathrm{P}$ are illustrated in Figure 4. The statistical comparisons for $\mathrm{Ca} / \mathrm{P}$ are summarized in the legend of Figure 4.

For females, statistically significant differences in $\mathrm{Ca} / \mathrm{P}$ were observed among most of the experimental groups. No statistical differences were found only between G3 (f-ovxad) and G6 (f-sham-ad), representing groups with ad libitum diets, which also demonstrated higher values of $\mathrm{Ca} / \mathrm{P}$.

For males, statistically significant differences were also observed among most of the experimental groups. No difference was observed between G12 (m-sham-ad) and G9 (morx-ad) nor between G12 (m-sham-ad) and G11 (m-shamiso). These groups (G9, G11, and G12) had the highest values of $\mathrm{Ca} / \mathrm{P}$.

For both females and males, all groups with the alcoholic diet (G1, G4, G7, and G10) had a lower Ca/P compared to groups with the ad libitum diet (G3, G6, G9, and G12). The lowest values of $\mathrm{Ca} / \mathrm{P}$ were found in groups subjected to the alcoholic diet in conjunction with hormonal deficiency: G1 (f-ovx-alc) for females and G7 (m-orx-alc) for males. These groups were used to test the hypothesis of this study.

\section{Discussion}

Our results suggest that diet had an important role, since the groups with ad libitum diet gained more weight in both females and males. However, factors related to gender also seem to have influenced the results, since all females gained weight during the experiment but some males showed weight reduction. This gender difference was previously observed by Jack Wallen et al. [25], who noticed that the deficiency of sex hormones reduced weight in males but increased it in females. Our study also showed that weight gain (in absolute values) was even more evident in females with estrogen deficiency (Ovx) and ad libitum diet (G3). This is similar to what occurs in women with menopause when high prevalence of overweight/obesity is reported [26].

For males, feed efficiency values were proportional to caloric intake for all groups. On the other hand, females on the alcoholic diet presented high caloric intake but low feed efficiency. One possible explanation for these results could be the great difficulty females have in metabolizing alcohol. Female bodies have lower total water volume compared to males. Because of this, females present higher blood alcohol levels than males given the same alcohol consumption [27]. In addition, females present lower gastric metabolism with lower alcohol dehydrogenase (ADH) activity [28], as well as a reduction of gluconeogenesis [29].

The $\mathrm{Ca} / \mathrm{P}$ ratio seems to be more reliable for analysis of bone mineralization disorders than separate analyses of $\mathrm{Ca}$ and $\mathrm{P}$ [30]. In experiments that induce osteoporosis, a decrease in $\mathrm{Ca} / \mathrm{P}$ is expected compared to normal bone [4]. Despite the well-known negative effect of the lack of sex hormones on bone quality $[7,9]$, there was no alteration in the $\mathrm{Ca} / \mathrm{P}$ ratio when the sex hormone deficiency was assessed alone (without association with dietary restrictions), in the present study. This was verified by the results observed in ad libitum groups in which sex hormone deficiency did not alter $\mathrm{Ca} / \mathrm{P}$ ratio in both females and males; group $\mathrm{G} 3$ ( $\mathrm{f}$ ovx-ad) was not different from G6 (f-sham-ad), and G9 (morx-ad) was not different from G12 (m-sham-ad). However, the conjunction of sex hormone deficiency and alcoholic diet (G1 and G7) led to the lowest $\mathrm{Ca} / \mathrm{P}$ ratios for both females and males, which confirmed the initial hypothesis of our experiment. A previous study [19] observed a decrease of the $\mathrm{Ca} / \mathrm{P}$ ratio in the alveolar bone crest region of $\mathrm{Ovx}$ females receiving a $20 \%$ alcohol solution for 8 weeks (the same experimental protocol of this study). In another study, Callaci et al. [18] observed vertebral bone loss in Ovx females receiving alcohol. This suggests that the deficiency of sex hormones associated with alcohol can affect different types of bones and is not restricted to the femur.

When comparing to groups with ad libitum diets, we observed a decreased $\mathrm{Ca} / \mathrm{P}$ ratio in all groups that ingested alcohol and in the majority of isocaloric groups, with the exception of the male group G11 (m-sham-iso), in which the highest $\mathrm{Ca} / \mathrm{P}$ ratio was found. These results suggest that the decrease in the $\mathrm{Ca} / \mathrm{P}$ ratio was evident for both females and males with an alcoholic diet. On the other hand, for groups with the isocaloric diet, the decrease in $\mathrm{Ca} / \mathrm{P}$ ratio was more evident in females. 
The use of isocaloric groups aimed to control for the nutritional problems related to a lower intake of food. Studies show that rats treated with alcohol ingest a significantly lower amount of solid food compared to groups with ad libitum diets $[19,21,22]$. This happens because alcohol is a substance with a low nutritional value but high energy content, which may lead to a feeling of satiety [15]. The use of isocaloric groups allows a control for the possible influence of decreased food intake on the results. It should be considered that an adequate intake of nutrients is important for the prevention and management of osteoporosis [16].

However, alcohol causes other important metabolic changes that were not controlled in this experiment. Alcohol consumption can lead to changes in the mucosa of the gastrointestinal tract, increased transport of toxins, and maldigestion and malabsorption of nutrients [31]. Alcohol also alters the metabolism of nutrients since it is a toxic substance which cannot be stored, and so alcohol must be oxidized preferentially to other nutrients [32]. Furthermore, alcohol causes various hormonal disorders involving secretions of several glands such as the hypothalamus, anterior pituitary, adrenal cortex, testicles, ovaries, thyroid gland, and pancreas, which could cause physiological and behavioral disorders [33]. The literature also reports that excessive alcohol consumption can lead to a decrease in vitamin $\mathrm{D}$ levels [34] which could impair calcium absorption [35]. It has been suggested that low levels of vitamin $\mathrm{D}$ in combination with low levels of sex steroid hormones considerably increase the risk of bone fractures [36].

In addition to the indirect effects of alcohol on bone metabolism causing important nutritional and hormonal changes, alcohol can also directly influence the activity of bone cells. The decrease in bone mass induced by alcohol consumption can be attributed to an imbalance between bone formation and bone resorption, with a predominant decrease in bone formation [13]. Rosa et al. [14] observed that alcohol intake affected the osteoblast function (important cells for bone formation and mineralization) in cell culture, inhibiting the bone matrix mineralization process. Similarly, a change in the pattern of bone mineralization (characterized by a decrease in the $\mathrm{Ca} / \mathrm{P}$ ratio) was observed in animals of alcohol groups (when compared to ad libitum groups), in the present experiment.

We observed that the alcoholic diet groups showed a state of lethargy and excessive rest, which may also have influenced the results since exercise can have a preventive effect on bone loss [37]. Therefore, it is not possible to know whether the results obtained in the alcohol groups are due to metabolic effects of alcohol, the state of lethargy and excessive resting (decreasing the physical activity), or both.

Another factor that must be considered is that the alcohol groups ingested the least amount of water, since dehydration could theoretically cause changes in the structure of hydroxyapatite $[38,39]$.

Some differences between males and females should be emphasized. The decrease of the $\mathrm{Ca} / \mathrm{P}$ ratio found in Orx males subjected to alcohol consumption (G7) was influenced mainly by a significant increase in phosphorus. On the other hand, in Ovx females subjected to alcohol consumption (G1) the decrease of the $\mathrm{Ca} / \mathrm{P}$ ratio was influenced mainly by a significant decrease in $\mathrm{Ca}$. The literature reports that both $\mathrm{Ca}$ and $\mathrm{P}$ are important nutrients for maintaining bone health [16]. However, it has also been suggested that an excess of phosphorus can impair bone health and be a risk factor for fractures [40].

This study has some limitations. The bone analysis is related only to its cortical area, since the X-ray coming from the rhodium tube does not penetrate deep into the sample. Therefore, there was no data related to trabecular bone. The loss of cortical and trabecular bone is important variable in assessing the risk of osteoporotic fractures [41]. An evaluation of trabecular bone could be considered in future studies.

In this study, only $\mathrm{Ca}$ and $\mathrm{P}$ were directly measured by the equipment. The organic elements were not directly measured because the equipment used in this study was not able to perform measurements for chemical elements below $\mathrm{Na}$ in atomic number. This fact can be considered a limitation of this study, since the bone inorganic phase consists of poorly crystalline carbonated apatite [1, 42]. Minerals are the main components of bone tissue, but not the only ones. Bone tissue is composed of minerals (50-70\%), organic matrix (20-40\%), water (5-10\%), and lipids (<3\%) [1]. A disease like osteoporosis is complex and can affect mineral and nonmineral components [43]. Other techniques such as Infrared and Raman spectroscopy could provide qualitative and quantitative information about the mineral and organic components of bone tissue and could be used in future studies [44].

It was previously suggested that, after comparison with other studies, the intake of alcohol by rats at a concentration of $20 \%$ for 8 weeks led to alcohol levels similar to that of animals having chronic and excessive alcohol consumption [19]. However, this comparison should be viewed with caution, since it would necessitate assessments of blood alcohol levels to have reliability. The lack of assessment of blood alcohol levels can be considered another limitation of the present study.

\section{Conclusions}

The present study demonstrated that 8 weeks of consuming $20 \%$ alcoholic solution reduced the $\mathrm{Ca} / \mathrm{P}$ ratio in the femurs of both female and male rats. The isocaloric diet reduced the $\mathrm{Ca} / \mathrm{P}$ ratio in females. In groups with ad libitum diet, the deficiency of sex hormones did change the $\mathrm{Ca} / \mathrm{P}$ ratio in females and males. However, the deficiency of sex hormones in conjunction with an alcoholic diet promoted the lowest values for the $\mathrm{Ca} / \mathrm{P}$ ratio in both females and males.

In short, one can conclude that there was a reduction in the $\mathrm{Ca} / \mathrm{P}$ ratio in the femur of both male and female rats who consumed alcohol and that this reduction was exacerbated when combined with a deficiency of sex hormones.

\section{Ethical Approval}

This study was performed in accordance with the ethical standards adopted in animal experimentation and was approved 
by the Ethics Committee of São José dos Campos School of Dentistry, Universidade Estadual Paulista (UNESP) (Protocol no. 037/2007-PA/CEP). All applicable institutional and/or national guidelines for the care and use of animals were followed.

\section{Competing Interests}

Karina Bortolin Lodi, Adriana Mathias Pereira da Silva Marchini, Ana Maria do Espírito Santo, Sigmar de Mello Rode, Leonardo Marchini, and Rosilene Fernandes da Rocha declare that they have no competing interests related to this study.

\section{Acknowledgments}

The authors acknowledge financial support from National Council for Scientific and Technological Development (CNPq-Brazil). The authors would also like to thank Ms. Anna Okulist for proofreading this paper. Karina Bortolin Lodi received a scholarship from the Brazilian governmental research agency, National Council for Scientific and Technological Development (CNPq-Brazil), to do this work.

\section{References}

[1] B. Clarke, "Normal bone anatomy and physiology," Clinical Journal of the American Society of Nephrology, vol. 3, supplement 3, pp. S131-S139, 2008.

[2] S. M. Moe, "Disorders involving calcium, phosphorus, and magnesium," Primary Care: Clinics in Office Practice, vol. 35, no. 2, pp. 215-237, 2008.

[3] P. J. Meunier and G. Boivin, "Bone mineral density reflects bone mass but also the degree of mineralization of bone: therapeutic implications," Bone, vol. 21, no. 5, pp. 373-377, 1997.

[4] N. Kourkoumelis, I. Balatsoukas, and M. Tzaphlidou, "Ca/P concentration ratio at different sites of normal and osteoporotic rabbit bones evaluated by Auger and energy dispersive X-ray spectroscopy," Journal of Biological Physics, vol. 38, no. 2, pp. 279-291, 2012.

[5] D. L. Glaser and F. S. Kaplan, "Osteoporosis. Definition and clinical presentation," Spine, vol. 22, no. 24, pp. 12s-16s, 1997.

[6] U. H. Lerner, "Bone remodeling in post-menopausal osteoporosis," Journal of Dental Research, vol. 85, no. 7, pp. 584-595, 2006.

[7] A. Falahati-Nini, B. L. Riggs, E. J. Atkinson, W. M. O’Fallon, R. Eastell, and S. Khosla, "Relative contributions of testosterone and estrogen in regulating bone resorption and formation in normal elderly men," Journal of Clinical Investigation, vol. 106, no. 12, pp. 1553-1560, 2000.

[8] B. L. Riggs, S. Khosla, and L. J. Melton III, "Sex steroids and the construction and conservation of the adult skeleton," Endocrine Reviews, vol. 23, no. 3, pp. 279-302, 2002.

[9] D. H. A. De Oliveira, T. M. Fighera, L. C. Bianchet, C. A. M. Kulak, and J. Kulak, "Androgens and bone," Minerva Endocrinologica, vol. 37, no. 4, pp. 305-314, 2012.

[10] S. Khosla, M. J. Oursler, and D. G. Monroe, "Estrogen and the skeleton," Trends in Endocrinology and Metabolism, vol. 23, no. 11, pp. 576-581, 2012.

[11] J. Chow, J. H. Tobias, K. W. Colston, and T. J. Chambers, "Estrogen maintains trabecular bone volume in rats not only by suppression of bone resorption but also by stimulation of bone formation," The Journal of Clinical Investigation, vol. 89, no. 1, pp. 74-78, 1992.

[12] P. Mikosch, "Alcohol and bone," Wiener Medizinische Wochenschrift, vol. 164, no. 1-2, pp. 15-24, 2014.

[13] D. B. Maurel, N. Boisseau, C. L. Benhamou, and C. Jaffre, "Alcohol and bone: review of dose effects and mechanisms," Osteoporosis International, vol. 23, no. 1, pp. 1-16, 2012.

[14] M. L. Rosa, M. M. Beloti, N. Prando, R. H. C. Queiroz, P. T. de Oliveira, and A. L. Rosa, "Chronic ethanol intake inhibits in vitro osteogenesis induced by osteoblasts differentiated from stem cells," Journal of Applied Toxicology, vol. 28, no. 2, pp. 205211, 2008.

[15] C. S. Lieber, "Alcohol and the liver: metabolism of alcohol and its role in hepatic and extrahepatic diseases," Mount Sinai Journal of Medicine, vol. 67, no. 1, pp. 84-94, 2000.

[16] R. Rizzoli, C. Abraham, and M.-L. Brandi, "Nutrition and bone health: turning knowledge and beliefs into healthy behaviour," Current Medical Research and Opinion, vol. 30, no. 1, pp. 131-141, 2014.

[17] K. N. Kelly and C. Kelly, "Pattern and cause of fractures in patients who abuse alcohol: what should we do about it?" Postgraduate Medical Journal, vol. 89, no. 1056, pp. 578-583, 2013.

[18] J. J. Callaci, D. Juknelis, A. Patwardhan, and F. H. Wezeman, "Binge alcohol treatment increases vertebral bone loss following ovariectomy: compensation by intermittent parathyroid hormone," Alcoholism: Clinical and Experimental Research, vol. 30, no. 4, pp. 665-672, 2006.

[19] A. M. P. S. Marchini, C. P. Deco, K. B. Lodi, L. Marchini, A. M. E. Santo, and R. F. Rocha, "Influence of chronic alcoholism and oestrogen deficiency on the variation of stoichiometry of hydroxyapatite within alveolar bone crest of rats," Archives of Oral Biology, vol. 57, no. 10, pp. 1385-1394, 2012.

[20] M. C. M. Salgado, A. M. P. D. Marchini, T. D. Tera, R. F. da Rocha, and L. Marchini, "Effects of estrogen deficiency combined with chronic alcohol consumption on rat mandibular condyle," Brazilian Journal of Oral Sciences, vol. 14, no. 1, pp. 1622, 2015.

[21] C. P. de Deco, A. M. P. da Silva Marchini, M. A. M. Bárbara, L. M. R. de Vasconcellos, R. F. da Rocha, and L. Marchini, "Negative effects of alcohol intake and estrogen deficiency combination on osseointegration in a rat model," Journal of Oral Implantology, vol. 37, no. 6, pp. 633-639, 2011.

[22] A. M. P. D. S. Marchini, L. L. Gonçalves, M. C. M. Salgado et al., "Alcoholic and isocaloric diet, but not ovariectomy, influence the apoptosis of bone cells within the alveolar bone crest of rats," Archives of Oral Biology, vol. 59, no. 4, pp. 424-433, 2014.

[23] F. M. Pascon, R. M. Puppin-Rontani, K. R. Kantovitz et al., "Morphological and chemical changes in dentin after using endodontic agents: fourier transform Raman spectroscopy, energy-dispersive X-ray fluorescence spectrometry, and scanning electron microscopy study," Journal of Biomedical Optics, vol. 17, no. 7, Article ID 075008, 2012.

[24] L. E. S. Soares, A. M. do Espírito Santo, A. Brugnera, F. A. N. A. Zanin, and A. A. Martin, "Effects of Er:YAG laser irradiation and manipulation treatments on dentin components, part 2: energy-dispersive X-Ray Fluorescence Spectrometry study," Journal of Biomedical Optics, vol. 14, no. 2, Article ID 024002, 2009.

[25] W. Jack Wallen, M. P. Belanger, and C. Wittnich, "Body weight and food intake profiles are modulated by sex hormones and 
tamoxifen in chronically hypertensive rats," Journal of Nutrition, vol. 132, no. 8, pp. 2246-2250, 2002.

[26] Y. Barrios Ospino, N. Díaz, L. Meertens et al., "Relation between leptin serun with weight and body fat distribution in postmenopausal women," Nutricion Hospitalaria, vol. 25, no. 1, pp. 80-84, 2010.

[27] A. L. Mirand and J. W. Welte, "Total body water adjustment of mean alcohol intakes," Journal of Substance Abuse, vol. 6, no. 4, pp. 419-425, 1994.

[28] E. Baraona, C. S. Abittan, K. Dohmen et al., "Gender differences in pharmacokinetics of alcohol," Alcoholism: Clinical and Experimental Research, vol. 25, no. 4, pp. 502-507, 2001.

[29] K. D. Sumida, A. A. Cogger, and A. V. Matveyenko, "Alcoholinduced suppression of gluconeogenesis is greater in ethanol fed female rat hepatocytes than males," Alcohol, vol. 41, no. 2, pp. 67-75, 2007.

[30] V. Zaichick and M. Tzaphlidou, "Calcium and phosphorus concentrations and the calcium/phosphorus ratio in trabecular bone from the femoral neck of healthy humans as determined by neutron activation analysis," Applied Radiation and Isotopes, vol. 58, no. 6, pp. 623-627, 2003.

[31] R. Rajendram and V. R. Preedy, "Effect of alcohol consumption on the gut," Digestive Diseases, vol. 23, no. 3-4, pp. 214-221, 2005.

[32] Y. Schutz, "Role of substrate utilization and thermogenesis on body-weight control with particular reference to alcohol," Proceedings of the Nutrition Society, vol. 59, no. 4, pp. 511-517, 2000.

[33] N. Rachdaoui and D. K. Sarkar, "Effects of alcohol on the endocrine system," Endocrinology and Metabolism Clinics of North America, vol. 42, no. 3, pp. 593-615, 2013.

[34] J. Alvisa-Negrín, E. González-Reimers, F. Santolaria-Fernández et al., "Osteopenia in alcoholics: effect of alcohol abstinence," Alcohol and Alcoholism, vol. 44, no. 5, pp. 468-475, 2009.

[35] P. P. Sirichakwal, A. Kamchansuppasin, C. C. Akoh, W. Kriengsinyos, S. Charoenkiatkul, and K. O. O'Brien, "Vitamin $\mathrm{D}$ status is positively associated with calcium absorption among postmenopausal Thai women with low calcium intakes," Journal of Nutrition, vol. 145, no. 5, pp. 990-995, 2015.

[36] J. A. Cauley, "Estrogen and bone health in men and women," Steroids, vol. 99, pp. 11-15, 2015.

[37] D. B. Maurel, N. Boisseau, S. Pallu, G. Y. Rochefort, C.-L. Benhamou, and C. Jaffre, "Regular exercise limits alcohol effects on trabecular, cortical thickness and porosity, and osteocyte apoptosis in the rat," Joint Bone Spine, vol. 80, no. 5, pp. 492498, 2013.

[38] P. Zhu, J. Xu, N. Sahar, M. D. Morris, D. H. Kohn, and A. Ramamoorthy, "Time-resolved dehydration-induced structural changes in an intact bovine cortical bone revealed by solid-state NMR spectroscopy," Journal of the American Chemical Society, vol. 131, no. 47, pp. 17064-17065, 2009.

[39] P. K. M. Tung, S. Mudie, and J. E. Daniels, "Hydration and radiation effects on the residual stress state of cortical bone," Acta Biomaterialia, vol. 9, no. 12, pp. 9503-9507, 2013.

[40] M. S. Calvo and K. L. Tucker, "Is phosphorus intake that exceeds dietary requirements a risk factor in bone health?" Annals of the New York Academy of Sciences, vol. 1301, no. 1, pp. 29-35, 2013.

[41] E. M. Stein, A. Kepley, M. Walker et al., "Skeletal structure in postmenopausal women with osteopenia and fractures is characterized by abnormal trabecular plates and cortical thinning," Journal of Bone and Mineral Research, vol. 29, no. 5, pp. 11011109, 2014.
[42] N. Kourkoumelis and M. Tzaphlidou, "Spectroscopic assessment of normal cortical bone: differences in relation to bone site and sex," TheScientificWorldJournal, vol. 10, pp. 402-412, 2010.

[43] Z. X. Wang, A. A. Lloyd, J. C. Burket, S. Gourion-Arsiquaud, and E. Donnelly, "Altered distributions of bone tissue mineral and collagen properties in women with fragility fractures," Bone, vol. 84, pp. 237-244, 2016.

[44] S. Gamsjaeger, R. Mendelsohn, A. L. Boskey, S. GourionArsiquaud, K. Klaushofer, and E. P. Paschalis, "Vibrational spectroscopic imaging for the evaluation of matrix and mineral chemistry," Current Osteoporosis Reports, vol. 12, no. 4, pp. 454464, 2014. 


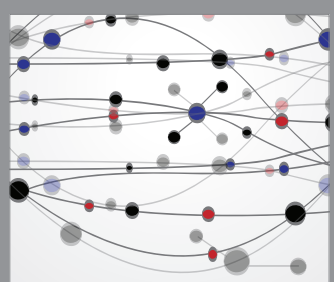

The Scientific World Journal
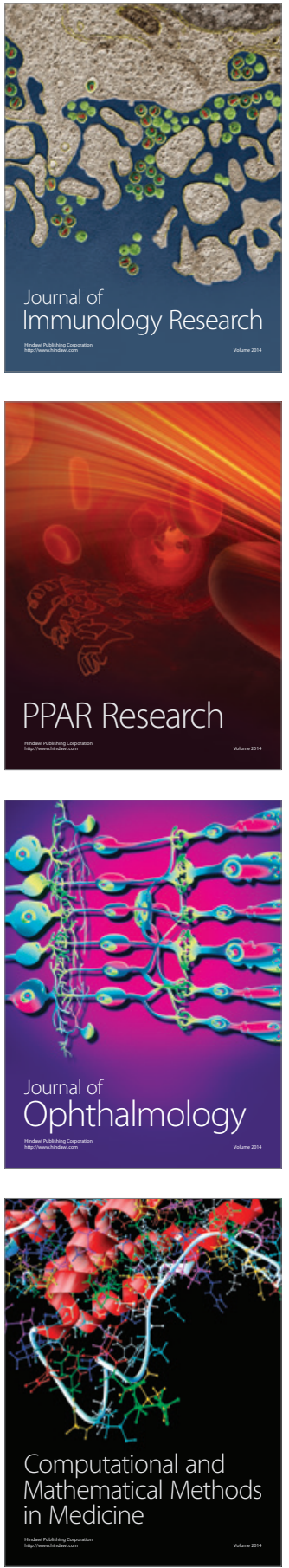

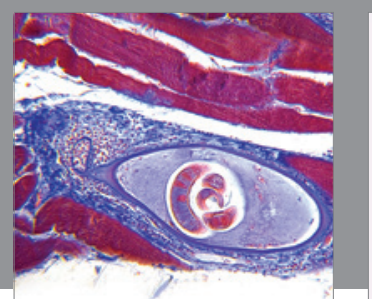

Gastroenterology Research and Practice

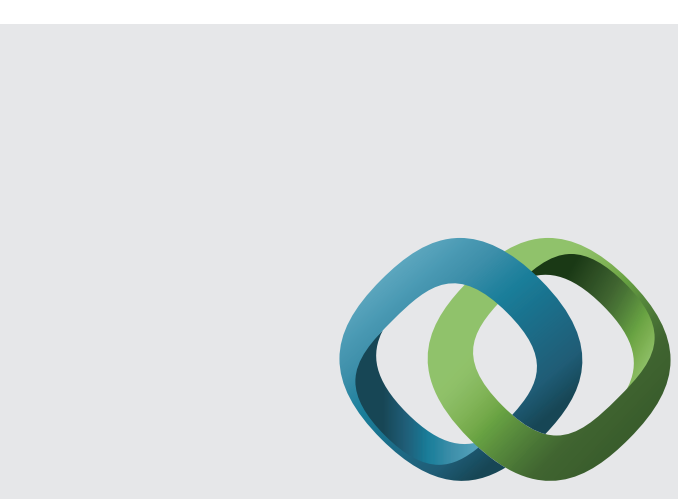

\section{Hindawi}

Submit your manuscripts at

http://www.hindawi.com
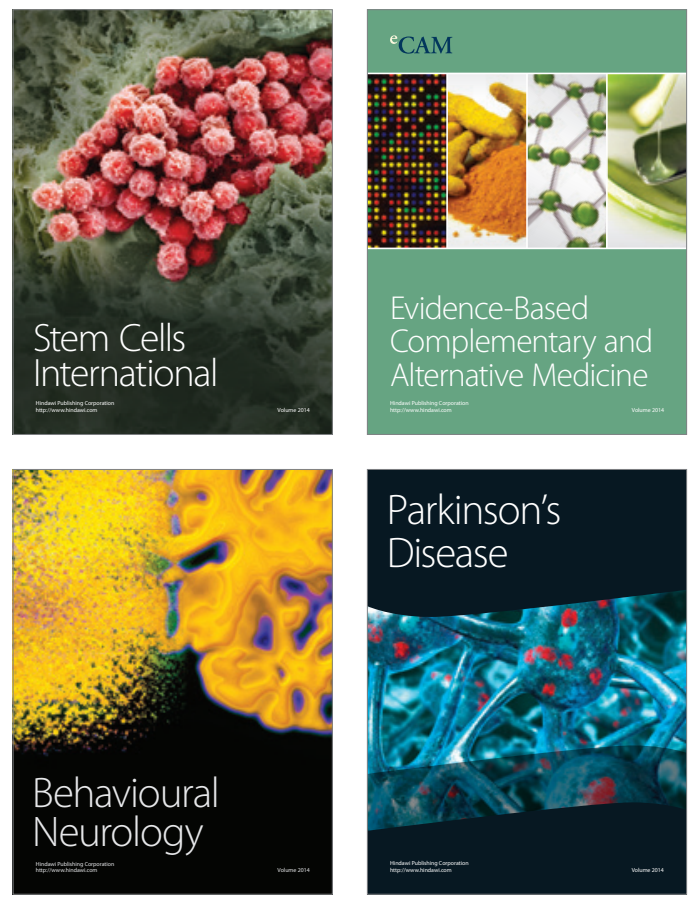
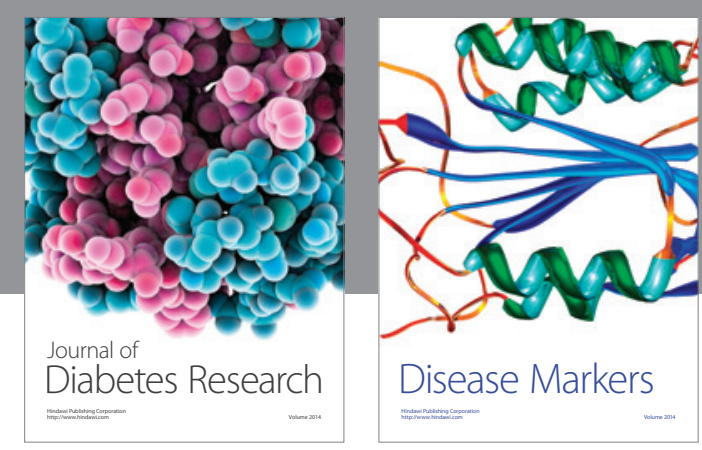

Disease Markers
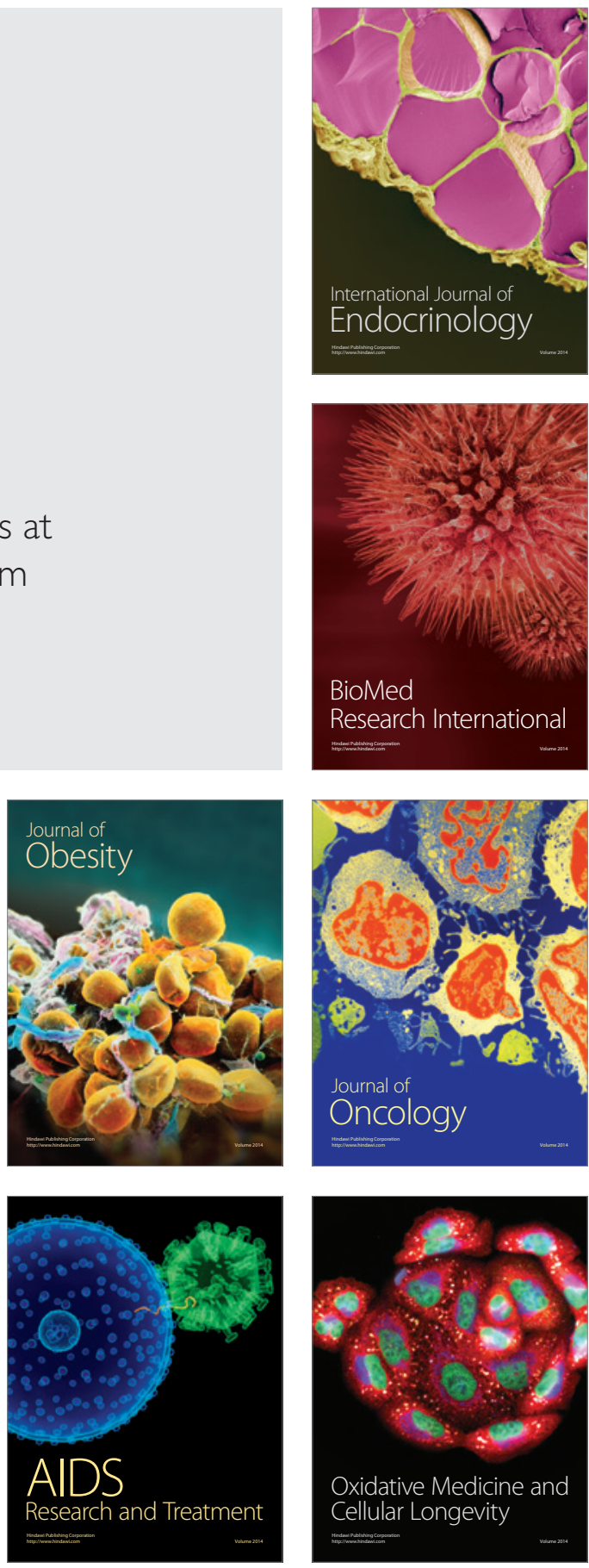